\begin{tabular}{|c|l|}
\hline Title & Calorimetric measurements of the phase transition in Rochelle salt-ammonium Rochelle salt mixed crystals \\
\hline Author(s) & Noda, N.; Nozaki, R.; Shiozaki, Y. \\
\hline Citation & $\begin{array}{l}\text { Physical Review B, 62(18), 12040-12044 } \\
\text { https://doi.org/10.1103/PhysRevB.62.12040 }\end{array}$ \\
\hline Issue Date & 2000-11-01 \\
\hline Doc URL & http://hdl.handle.net/2115/56815 \\
\hline Rights & ○ 2000 The American Physical Society \\
\hline Type & article \\
\hline File Information & PhysRevB.62.12040.pdf \\
\hline
\end{tabular}

Instructions for use 


\title{
Calorimetric measurements of the phase transition in Rochelle salt-ammonium Rochelle salt mixed crystals
}

\author{
N. Noda, R. Nozaki, and Y. Shiozaki \\ Division of Physics, Graduate School of Science, Hokkaido University, Sapporo 060-0810, Japan
}

(Received 13 March 2000)

\begin{abstract}
The specific heats, which are given with absolute value, of Rochelle salt-ammonium Rochelle salt mixed crystals $\left(\mathrm{RS}_{1-x}-\mathrm{ARS}_{x}\right)$ in a region $0.18 \leqslant x \leqslant 0.9$ have been measured between about $10 \mathrm{~K}$ and $300 \mathrm{~K}$ by an ac calorimetric method. A very small anomaly was found around the transition for any $x$. It becomes larger as $x$ increases. The anomalies have been well described using the Landau-type free energy expanded to the sixth order of polarization. It was found that, as $x$ increases, the transition point approaches the tricritical point at which a crossover from the second-order phase transition to the first-order one takes place. Transition entropy has been calculated from the anomalous specific heat. It increases from about $8 \%$ to about $55 \%$ of $R \ln 2$ with increasing $x$ in the measured region. Very small values for small $x$ in this region suggest the displacivelike character in the phase transition. And the order-disorder motion contributes more and more to the phase transition with increasing $x$.
\end{abstract}

\section{INTRODUCTION}

Rochelle salt $\left(\mathrm{NaKC}_{4} \mathrm{H}_{4} \mathrm{O}_{6} \cdot 4 \mathrm{H}_{2} \mathrm{O}\right.$, abbreviated to RS) is a well-known ferroelectric. It has an unusual feature of the phase transition: After the paraelectric-ferroelectric phase transition, the ferroelectric phase changes to a paraelectric phase again with decreasing temperature. Ammonium Rochelle salt $\left(\mathrm{NaNH}_{4} \mathrm{C}_{4} \mathrm{H}_{4} \mathrm{O}_{6} \cdot 4 \mathrm{H}_{2} \mathrm{O}\right.$, ARS), which is isomorphous to RS in the paraelectric state, undergoes a characteristic phase transition, but its feature is very different from that of RS. ARS is often called the improper ferroelectric, because the order parameter of the phase transition is not the polarization though spontaneous polarization exists in the low-temperature phase. The spontaneous polarization appears in the direction of the $b$ axis in contrast with the $a$ axis for RS. The phase transition of ARS is of first order, while those of RS are of second order. It is very interesting that considerable changes in the phase transition thus take place only by replacing $\mathrm{K}^{+}$in $\mathrm{RS}$ with $\mathrm{NH}_{4}^{+}$in ARS.

Mixed crystals of RS and ARS $\left[\mathrm{NaK}_{1-x}\left(\mathrm{NH}_{4}\right)_{x} \mathrm{C}_{4} \mathrm{H}_{4} \mathrm{O}_{6}\right.$ - $\left.4 \mathrm{H}_{2} \mathrm{O}, \mathrm{RS}_{1-x}-\mathrm{ARS}_{x}\right]$ can be formed in whole range of $x$ $(0 \leqslant x \leqslant 1)$. The RS-ARS mixed crystal system is divided into four regions according to the dielectric properties. ${ }^{1}$ In region I $(0 \leqslant x \leqslant 0.025)$, the mixed crystals undergo two successive phase transitions and a ferroelectric phase with polar $a$ axis appears between two transition temperatures, as the same with RS. In region II $(0.025 \leqslant x \leqslant 0.18)$, no phase transition occurs. In region III $(0.18 \leqslant x \leqslant 0.9)$, only one phase transition, which is proper ferroelectric, occurs again. The low-temperature phase is of ferroelectric along the $a$ axis. The mixed crystals in region IV $(0.9 \leqslant x \leqslant 1)$ undergo an improper ferroelectric phase transition as ARS and are polar along the $b$ axis in the low-temperature phase.

It is very important to study $\mathrm{RS}_{1-x}$ - $\mathrm{ARS}_{x}$ mixed crystals as a function of $x$ for understanding the reasons for the difference of the phase transition between RS and ARS. The dielectric properties in this system have been much studied by many workers. ${ }^{1-7}$ From measurements of the complex dielectric constants for RS, Debye-type dielectric relaxation with critical slowing down was observed in the microwave frequency range by Sandy and Jones ${ }^{5}$ and the existence of the soft mode was pointed out by Volkov et al. ${ }^{6}$ from behavior in the millimeter to submillimeter frequency range. The former indicates the order-disorder phase transition; the latter indicates the displacive one. Horioka et al. ${ }^{7}$ measured the complex dielectric constant in the microwave frequency range for the mixed crystals in region III. They reported similar results to Sandy and Jones for RS. It suggests the order-disorder phase transition in region III.

The specific heat is one of the most important and basic quantities for the study of phase transitions. Nevertheless, the study of the specific heat is rarely reported for the RSARS mixed crystal system except for RS. The specific heat of RS exhibits extremely small anomalies at the transitions. ${ }^{8,9}$ In our previous measurements,${ }^{10}$ the specific heat of ARS shows a very large, sharp peak at the transition. It is thought that the difference of the specific heat anomaly between RS and ARS reflects the difference of their phase transitions. Then it is expected that the $x$ dependence of the specific heat anomaly brings valuable information on the change of the phase transition. Moreover, the transition entropy, which is calculated from the specific heat anomaly, is known to give very important information on the phase transition mechanism. In the present study, we have precisely measured the specific heat and investigated the anomalous behavior as a function of $x$. We have focused on region III, since this region occupies the widest $x$ range.

\section{EXPERIMENT}

Single crystals of $\mathrm{RS}_{1-x}-\mathrm{ARS}_{x}$ were grown in aqueous solutions by the cooling method. Since the concentration of ammonium ion contained in the crystal, which is represented by $x$, is generally different from that in the mother solution, the values of $x$ were decided from the relation between them determined by Makita and Takagi ${ }^{1}$ and Shiozaki et al. ${ }^{11}$ The samples used in the measurements were prepared about 2 $\times 2 \times 0.1 \mathrm{~mm}^{3}$ in size. The specific heat was measured by an ac calorimetric technique in a temperature range between 
about $10 \mathrm{~K}$ and $300 \mathrm{~K}$. The frequency of periodic heating was set to $3 \mathrm{~Hz}$. By observing a linear relation between the inverse of the frequency and amplitude of the sample temperature oscillation it was made sure that this frequency satisfied two conditions: the temperature gradient in the sample is disregarded and the thermal leak from the sample to the bath does not occur: These conditions, respectively, give the upper and the lower limits of the frequency range. Other detailed experimental conditions have been reported in our previous paper. ${ }^{10,12,13}$

In general, the absolute value of the specific heat is not obtained directly by the light-irradiation-type ac calorimetric method as ours because the amount of heat energy supplied to the sample is not known. In addition, the heat capacity contributed from additional materials attached on the sample face, varnish, carbon powder, etc., may not be neglected, because the sample size is very small. ${ }^{14}$ Then we determined the absolute value by measuring the supplied heat energy and the additional heat capacity. The process has already been reported in detail. ${ }^{13}$

\section{RESULTS AND INTERPRETATION}

The temperature dependences of the specific heat around the transition are shown in Fig. 1 for mixed crystals with $x$ $=0.27,0.62$, and 0.88 . An anomaly accompanied by the phase transition is found in each curve. The anomalies are very small, especially for $x=0.27$. The anomalous behavior for these crystals is similar to that for RS. ${ }^{8,9}$ However, it is not similar to that for ARS, which shows a very large, sharp peak at the transition. ${ }^{10}$ It is found that the anomaly becomes larger as $x$ increases. In Fig. 1, the specific heats appear to exhibit a tail in the high-temperature side of the transition. It is made sure that the behavior of the tail was different among the samples with the same $x$. A thermal hysteresis in the anomaly was not observed for any crystal.

In order to evaluate the specific heat accompanied by the phase transition in region III, the experimental data have been fitted with a function as

$$
c_{p}(T)=\sum_{n} k_{n} T^{n}+\Delta c_{p}(T) .
$$

The first term on the right-hand side of Eq. (1) corresponds to the background. It is considered to be a smooth function of temperature, so that it is expressed by the polynomial of temperature. The second term is the anomaly.

The expression of $\Delta c_{p}(T)$ has been given as follows: The specific heat anomaly is expected to be described within the mean field approximation, since Horioka et al. reported that the Curie-Weiss law is satisfied in region III. ${ }^{7}$ The tail which is seen in Fig. 1 is not considered to be ascribed to a critical phenomenon because if it is caused by the critical phenomenon, its behavior should not depend on the samples. In addition, in theory a mean field approximation is exactly valid for uniaxial and piezoelectric ferroelectrics. ${ }^{15-17}$ The mixed crystals in region III are just the uniaxial and piezoelectric ferroelectrics, because the spontaneous polarization appears in the direction of only the $a$ axis and the space group is $P 2{ }_{1} 2{ }_{1} 2$ in the paraelectric phase. Then the anomalous spe-
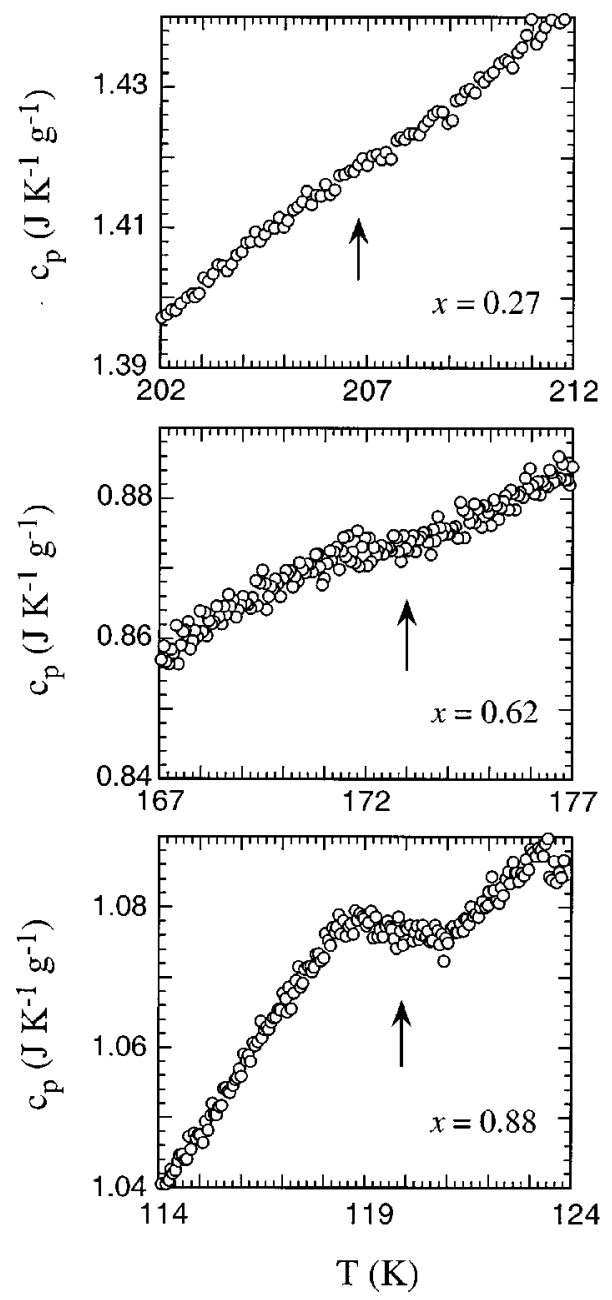

FIG. 1. Temperature dependences of the specific heat around the transition for the mixed crystals in region III. The arrows indicate the transition point.

cific heat was described by using the Landau-type free energy. The free energy is expanded to the sixth order of polarization as

$$
G=a t P^{2}+b P^{4}+c P^{6},
$$

where $t \equiv\left(T-T_{0}\right) / T_{0}$ is a reduced temperature and $T_{0}$ is the transition temperature. It is noted that the phase transition in region III is of second order. It turned out from preliminary fitting runs that the observed specific heat anomalies were able to be described very well by using the expansion of Eq. (2). The coefficients $a$ and $c$ are positive, and $b$ is also positive because of the second-order phase transition. ${ }^{18}$ Here, it has been assumed that the distribution of the transition temperature is caused by an inhomogeneity in the sample in order to describe the tail in high-temperature side of the transition. Because no thermal hysteresis was observed, it is hardly considered that a strain or a defect in the crystal affected the behavior around the transition. Moreover, an effect of impurities can be included in the distribution of the concentration. Then, using Eq. (2), the anomaly is written as

$$
\Delta c_{p}(T)=\int_{T}^{\infty} A \frac{T}{T_{0}}\left[1-\frac{3}{t_{0}}\left(\frac{T-T_{0}}{T_{0}}\right)\right]^{-1 / 2} g\left(T_{0}\right) d T_{0},
$$




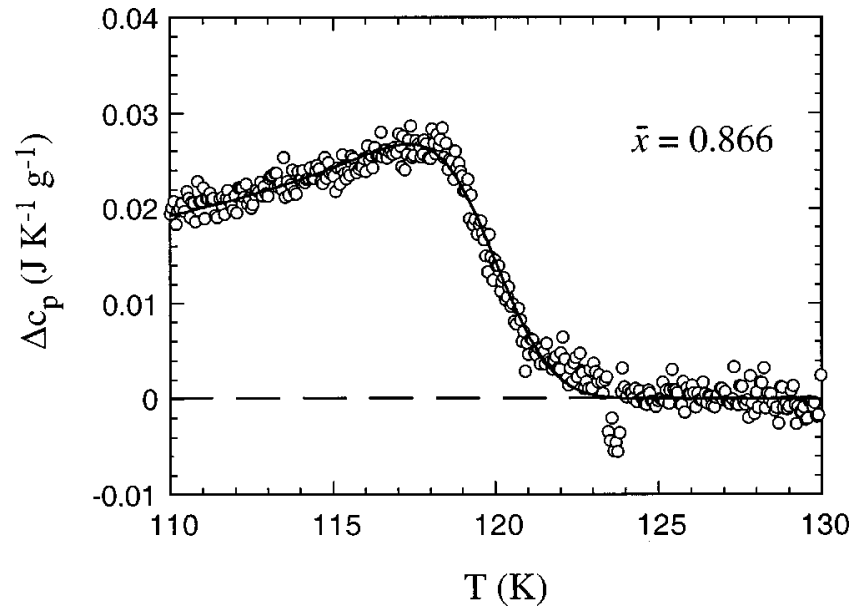

FIG. 2. Typical result of the fitting. The circles show observed data points. The solid line is the calculated curve obtained from the fitting.

where $A \equiv a^{2} / 2 b T_{0}$ and $t_{0} \equiv b^{2} / a c$. The distribution function $g\left(T_{0}\right)$ is given as

$$
g\left(T_{0}\right)=\frac{1}{\sqrt{2 \pi} \sigma} \exp \left[-\frac{\left(T_{0}-\bar{T}_{0}\right)^{2}}{2 \sigma^{2}}\right],
$$

with the mean value of the transition temperature $\bar{T}_{0}$ and the dispersion $\sigma^{2} \cdot 19$

A typical result of the fitting is shown in Fig. 2. It shows that the fit of the calculated result to the observed one is very good.

\section{DISCUSSION}

It has been shown that the anomalous behavior of the specific heat is well described by the model induced from the Landau-type free energy of Eq. (2) with the distribution of the transition temperature. Figure 3 shows anomalous spe-

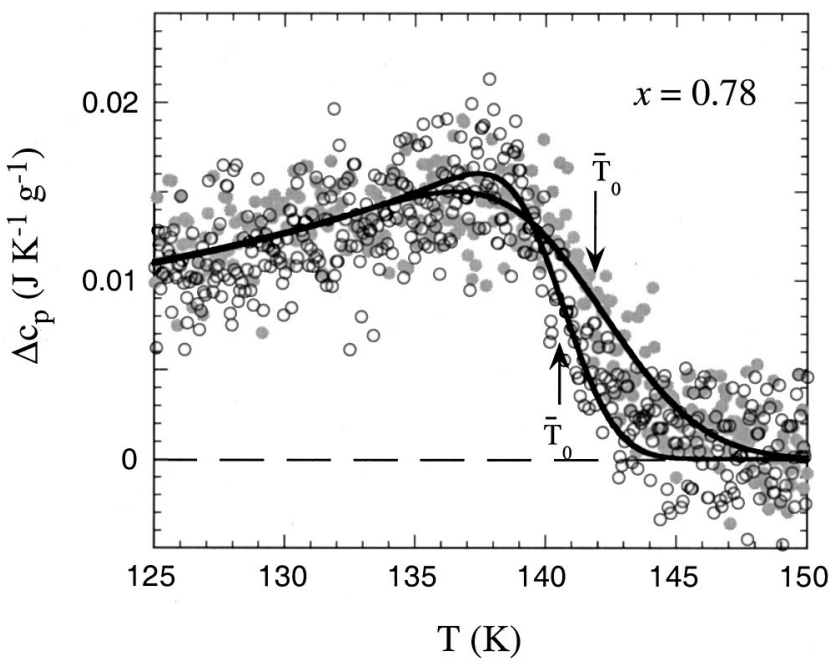

FIG. 3. Temperature dependences of the specific heat anomalies for two samples of the same $x$. The open and gray circles are experimental ones and the solid lines are the calculated ones. $\bar{T}_{0}$ is the mean value of the transition temperature.

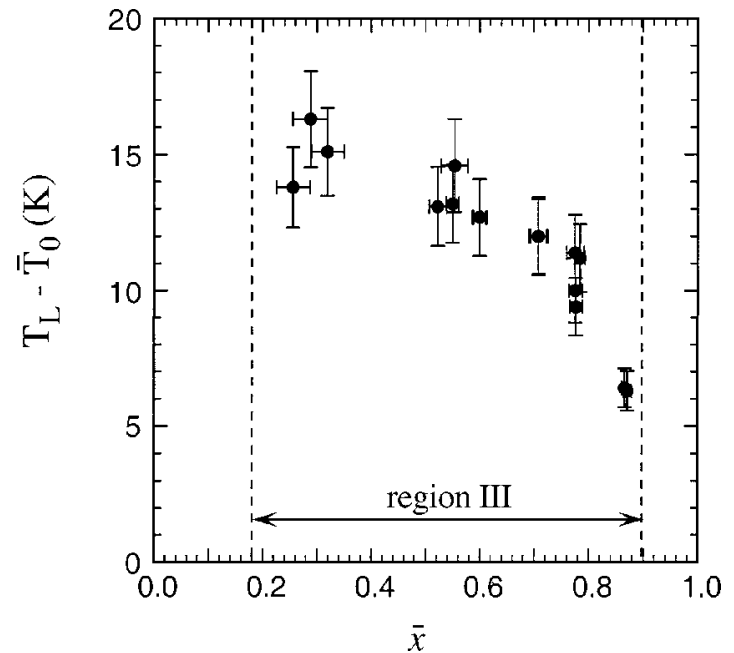

FIG. 4. Dependence of $T_{\mathrm{L}}-\bar{T}_{0}$ on $\bar{x}$ in region III. Error bars for the vertical axis are given by the distribution of the transition temperature. The error of concentration is determined from the distribution of the transition temperature.

cific heats for two samples which were cut out of one crystal block. The behaviors of the tails above the peak temperature are different from each other. The mean values of the transition temperature, $\bar{T}_{0}$, are also different. It shows that the distribution of the concentration varies with respect to the location in a block of the crystal. Then a mean value of the concentration for sample, $\bar{x}$, may not be identified with $x$, which represents the concentration of the crystal block. The value of $\bar{x}$ for each sample used in the measurements was determined from $\bar{T}_{0}$ by using the phase diagram of the RSARS mixed crystal system. ${ }^{1,11}$ The variation in $\bar{x}$ among the samples for the same $x$ is found to be 0.06 at most. The distribution of the concentration around $\bar{x}$ is not larger than the variation in the data of $x$ measured by several groups. Hereafter, we use $\bar{x}$ as the concentration of each sample.

The specific heat anomaly can be also expressed from the Landau-type free energy of Eq. (2) as

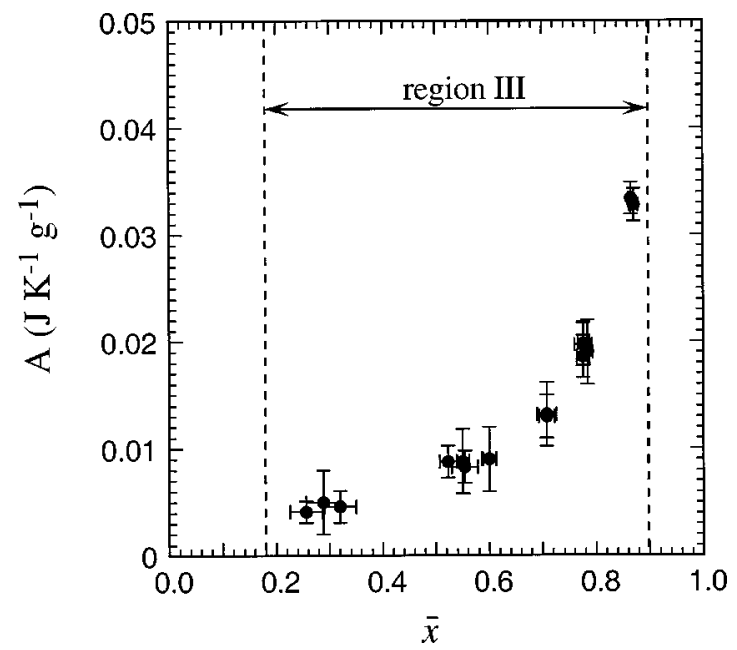

FIG. 5. $\bar{x}$ dependence of the excess specific heat at $\bar{T}_{0}$. Error bars for the vertical axis are given by the variation in the observed data points. 


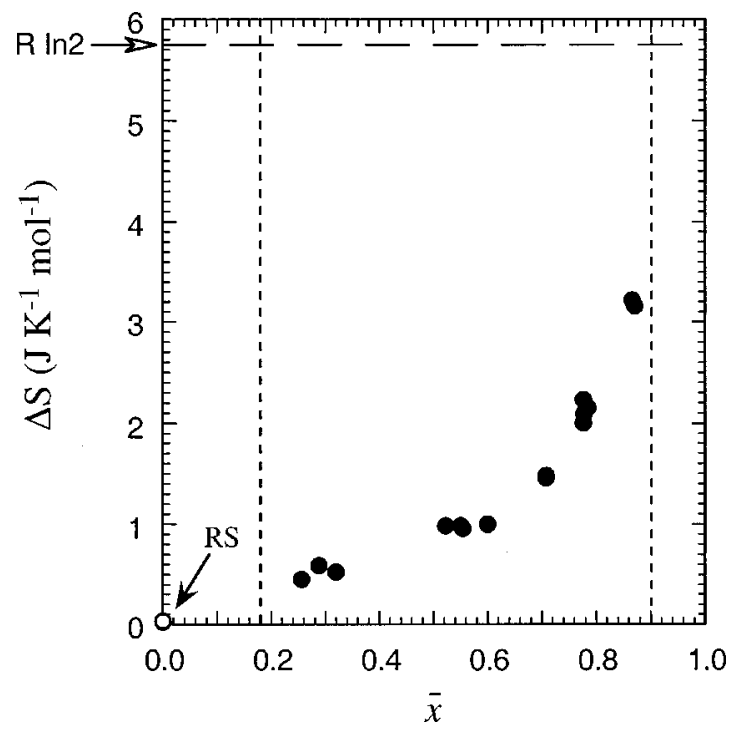

FIG. 6. Transition entropies in region III (solid circles). Open circles are the values for the upper and the lower transitions in RS obtained by Tatsumi et al. (Ref. 9).

$$
\Delta c_{p}(T)=A^{*} \frac{T}{T_{0}}\left(T_{\mathrm{L}}-T\right)^{-1 / 2}
$$

where

$$
T_{\mathrm{L}} \equiv\left(1+\frac{t_{0}}{3}\right) T_{0}=\left(1+\frac{b^{2}}{3 a c}\right) T_{0}
$$

and

$$
A^{*} \equiv A\left(T_{\mathrm{L}}-T_{0}\right)^{1 / 2}=\left(\frac{a^{3}}{12 c T_{0}}\right)^{1 / 2},
$$

as often successfully used in specific heat analyses with such free energy in the field of liquid crystals. ${ }^{19-21}$ The coefficient of the fourth-order term in the free energy, $b$, is positive for the second-order phase transition, negative for the first-order one, and zero for the tricritical point at which a crossover from second order to first order takes place. It is known from Eq. (6) that at the tricritical point $T_{\mathrm{L}}$ is identified with the transition temperature $T_{0}$. In the first-order and the secondorder phase transitions $(b \neq 0), T_{\mathrm{L}}$ is always larger than $T_{0}$, because $a$ and $c$ are positive. As shown in Fig. $4, T_{\mathrm{L}}-\bar{T}_{0}$ decreases with increasing $\bar{x}$ in region III. It indicates that the transition point approaches the tricritical point as $x$ increases. However, it is not known at present whether this tendency is connected with the first-order phase transition in region IV or not, because the transition mechanism in region IV is very different from that in region III. It is necessary for understanding the connection to study around the boundary of regions III and IV minutely.

Figure 5 shows the excess of the specific heat at the transition temperature, which was obtained from fitting of the experimental data, against $\bar{x}$. It is clarified that the excess specific heat at the transition temperature continuously increases with increasing $\bar{x}$. Integrating the excess specific heat divided by temperature in a wide temperature range, the transition entropy $\Delta S$ was calculated. In Fig. 6 the $\bar{x}$ dependence of $\Delta S$ is shown. It is very similar to the dependence of the

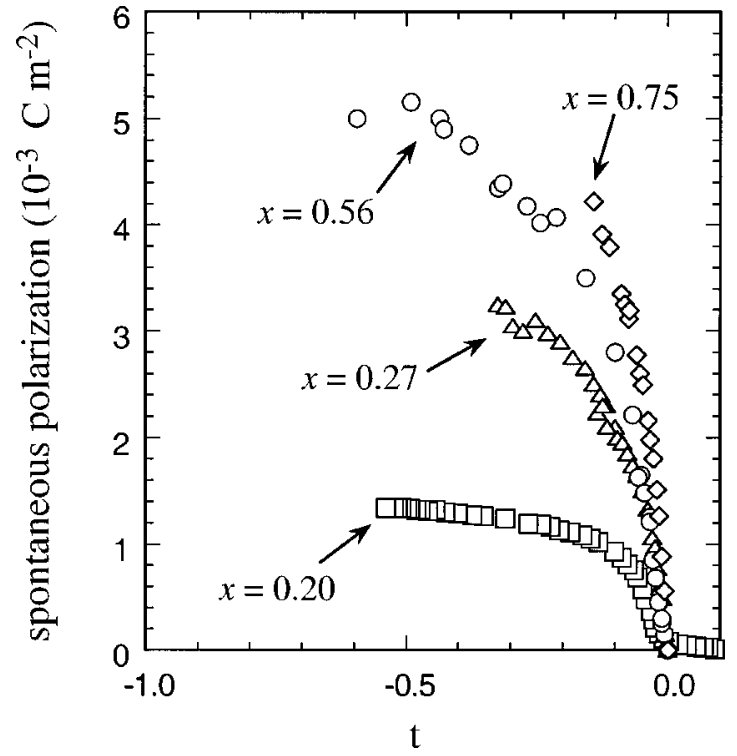

FIG. 7. Spontaneous polarization vs reduced temperature for various $x$ in region III (Ref. 3).

excess specific heat. It is found that the transition entropy continuously increases with increasing $\bar{x}$ and $\Delta S$ for $\bar{x}$ $\sim 0.88$ is about 10 times larger than that for $\bar{x} \sim 0.30$. This tendency is supported by the $x$ dependence of the saturated spontaneous polarization. ${ }^{3}$ The transition entropy and the saturated spontaneous polarization $P_{\mathrm{s}}$ are related by an equation

$$
\Delta S=\frac{a}{T_{0}} P_{\mathrm{s}}^{2}
$$

from the free energy expansion of Eq. (2). Figure 7 shows that $P_{\mathrm{s}}$ becomes larger with increasing $x$. So the tendency of increasing $\Delta S$ is indicated.

A unified model of the order-disorder and the displacive phase transitions is proposed by Onodera. ${ }^{22} \mathrm{He}$ discussed the difference between these transitions by the height of the potential barrier for dipolar motion: The height increases as the order-disorder character becomes strong and the displacive one becomes weak. It is considered from the $\bar{x}$ dependence of the transition entropy that the phase transition continuously changes in that sense from the displacive-side type to the order-disorder-side type with increasing $x$ in region III. Recent dielectric studies in the microwave frequency region by Oka et $a l .{ }^{23}$ suggest that the barrier between two the minima in the potential for dipoles increases with increasing $x$ in region III. This tendency agrees with our suggestion.

The values of $\Delta S$ at two transitions of RS obtained by Tatsumi et al. ${ }^{9}$ are also shown in Fig. 6. It is notable that they appear to be continuous with the $x$ dependence in region III. However, the reason for such a relation between RS and region III is not clear yet. It should be a future problem.

\section{ACKNOWLEDGMENTS}

We are thankful to Professor T. Yagi, Professor M. Tokunaga, and Professor K. Wada for valuable discussions. We would also like to thank Dr. H. Haga for continuous encouragement. 
${ }^{1}$ Y. Makita and Y. Takagi, J. Phys. Soc. Jpn. 13, 367 (1958).

${ }^{2}$ Hablützel, Helv. Phys. Acta 12, 489 (1939).

${ }^{3}$ Y. Shiozaki, H. Toki, H. Kabasawa, E. Suzuki and R. Nozaki, Ferroelectrics 184, 301 (1996).

${ }^{4}$ N.R. Ivanov, L.A. Shuvalov, D. Khusravov, and N.M. Shchagina, Kristallografiya 25, 1221 (1980) [Sov. Phys. Crystallogr. 25, 698 (1980)].

${ }^{5}$ F. Sandy and R.V. Jones, Phys. Rev. 168, 481 (1968).

${ }^{6}$ A.A. Volkov, G.V. Kozlov, E.B. Kryukova and J. Petzelt, Zh. Éksp. Teor. Fiz. 90, 192 (1986) [Sov. Phys. JETP 63, 110 (1986)].

${ }^{7}$ M. Horioka, R. Abe, and T. Naka, J. Phys. Soc. Jpn. 49, 599 (1980).

${ }^{8}$ A.J.C. Wilson, Phys. Rev. 54, 1103 (1938).

${ }^{9}$ M. Tatsumi, T. Matsuo, H. Suga, and S. Seki, J. Phys. Chem. Solids 39, 427 (1978).

${ }^{10}$ N. Noda, H. Haga, T. Kikuta, R. Nozaki, and Y. Shiozaki, Ferroelectrics 168, 169 (1995).

${ }^{11}$ Y. Shiozaki, T. Nishimura, A. Amano, E. Suzuki, and R. Nozaki, Ferroelectrics 158, 151 (1994).

${ }^{12}$ N. Noda, H. Haga, H. Nakano, R. Nozaki, and Y. Shiozaki, Fer- roelectrics 184, 293 (1996).

${ }^{13}$ N. Noda, H. Nakano, H. Haga, R. Nozaki, and Y. Shiozaki, J. Korean Phys. Soc. 29, S513 (1996).

${ }^{14}$ K. Ema, K. Hamano, K. Kurihara, and I. Hatta, J. Phys. Soc. Jpn. 43, 1954 (1977).

${ }^{15}$ T. Mitsui, E. Nakamura, and M. Tokunaga, Ferroelectrics 5, 185 (1973).

${ }^{16}$ A.I. Larkin and D.E. Khmel'nitskii, Zh. Éksp. Teor. Fiz. 56, 2087 (1969) [Sov. Phys. JETP 29, 1123 (1969)].

${ }^{17}$ A.P. Levanyuk and A.A. Sobyanin, Pis'ma Zh. Éksp. Teor. Fiz. 11, 540 (1970) [JETP Lett. 11, 371 (1970)].

${ }^{18}$ L.D. Landau and E.M. Lifshitz, Statistical Physics (Pergamon Press, London, 1959), Chap. 14, p. 452.

${ }^{19}$ T. Chan, Ch. Bahr, G. Heppke, and C.W. Garland, Liq. Cryst. 13, 667 (1993).

${ }^{20}$ J.B. Goates, C.W. Garland, and R. Shashidhar, Phys. Rev. A 41, 3192 (1990).

${ }^{21}$ C.C. Huang and J.M. Viner, Phys. Rev. A 25, 3385 (1982).

${ }^{22}$ Y. Onodera, Prog. Theor. Phys. 44, 1477 (1970).

${ }^{23}$ A. Oka, R. Nozaki, and Y. Shiozaki, Ferroelectrics 230, 157 (1999). 